\title{
Half the entanglement in critical systems is distillable from a single specimen
}

\author{
R. Orús ${ }^{1}$, J.I. Latorre ${ }^{1}$, J. Eisert ${ }^{2,3}$, and M. Cramer ${ }^{4}$ \\ 1 Dept. d'Estructura i Constituents de la Matèria, Univ. Barcelona, 08028, Barcelona, Spain \\ 2 QOLS, Blackett Laboratory, Imperial College London, Prince Consort Road, London SW7 2BW, UK \\ 3 Institute for Mathematical Sciences, Imperial College London, Exhibition Rd, London SW7 2BW, UK \\ 4 Institut für Physik, Universität Potsdam, Am Neuen Palais 10, D-14469 Potsdam, Germany
}

(Dated: August 16, 2018)

\begin{abstract}
We establish a quantitative relationship between the entanglement content of a single quantum chain at a critical point and the respective entropy of entanglement. We find that surprisingly, the leading critical scaling of the single-copy entanglement with respect to any bi-partitioning is exactly one half of the entropy of entanglement, in a general setting of conformal field theory and quasi-free systems. Conformal symmetry imposes that the single-copy entanglement scales as $E_{1}\left(\rho_{L}\right)=(c / 6) \log L-(c / 6)\left(\pi^{2} / \log L\right)+O(1 / L)$, where $L$ is the number of constituents in a block of an infinite chain and $c$ denotes the central charge. This shows that from a single specimen of a critical chain, already half the entanglement can be distilled compared to the rate that is asymptotically available. The result is substantiated by a quantitative analysis for all translationally invariant quantum spin chains corresponding to all isotropic quasi-free fermionic models. An example of the XY spin chain shows that away from criticality the above relation is only maintained near the quantum phase transition.
\end{abstract}

PACS numbers: 03.75.Ss, 03.75.Lm, 03.75.Kk

How much entanglement is contained in a many-body system at zero temperature? Variants of this question have received a significant attention in recent years, notably in the context of condensed matter systems [1, 2, 3, 4, 5, 6, 7, 8, 9, 10, 11, 12, 13, 14, 15, 16]. In particular, it has turned out that the scaling of entanglement quantities - similar to that of two-point correlators [17] - is indeed intimately intertwined with critical behavior, and that signatures of quantum phase transitions become manifest. In one-dimensional systems in particular, it has been found that criticality is typically accompanied with the entanglement of a subblock consisting of a number of consecutive constituents to be logarithmically divergent [3, 4, 5]. Such a behavior of the entropy of a subblock has also been linked to the performance of numerical DMRG-type simulations in many-body systems close to critical points [3, 15]. This quantity has a clearcut interpretation in entanglement theory: the entropy measures the degree of entanglement, in that it determines the optimal rate at which maximally entangled pairs can be distilled from a given state. Such a procedure may invoke any collective local quantum operations, assisted with classical communication (LOCC), under the assumption that one has infinitely many identically prepared spin systems at hand [18]. So in the present context, it would quantify the entanglement in this asymptotic sense, when operating locally on a subblock and the rest of the system, but on many identical systems.

Needless to say, one may equally reasonably ask: how much entanglement is contained in a single specimen of a many-body system? This is meant as the largest entanglement content that any apparatus could potentially distill with certainty from just one quantum chain at hand, resembling the situation that one would actually encounter in any experiment. More specifically: what is the largest dimension of a maximally entangled state - or, equivalently, the maximum number of maximally entangled qubit pairs - that can be distilled with certainty from a single specimen of a system with any physical device? The logarithm of this quan- tity, introduced in Ref. [16], will be referred to as single-copy entanglement. That is, for a state $\rho$ of a one-dimensional chain with reduction $\rho_{L}$ to a block consisting of $L$ consecutive constituents we write for the single-copy entanglement $E_{1}\left(\rho_{L}\right)=\log M$ if $\rho \longmapsto\left|\psi_{M}\right\rangle\left\langle\psi_{M}\right|$ under LOCC, where $\left|\psi_{M}\right\rangle=M^{-1 / 2} \sum_{i=1}^{M}|i, i\rangle$ [19]. Noting that single-copy transformation of pure states under LOCC is governed by a majorization relation to the reduced states [20], one finds that $E_{1}\left(\rho_{L}\right)=\log \left\lfloor 1 / \lambda_{1}\right\rfloor$, where $\lambda_{1}$ is the largest eigenvalue of the reduced state $\rho_{L}$ of a block of length $L$ [21].

In this work, we establish a fully quantitative relationship between this single-copy quantity and the geometric entropy - the entropy of entanglement - valid in a very large class of many-body systems at a critical point. More specifically, for a subblock of length $L$ we compare the single-copy entanglement $E_{1}\left(\rho_{L}\right)$ with the entropy of entanglement $S\left(\rho_{L}\right)=$ $-\operatorname{tr}\left[\rho_{L} \log \rho_{L}\right]$ [22]. We invoke the machinery of conformal field theory [24] and of quasi-free systems to relate these entanglement contents for a single specimen and the maximal asymptotically achievable rate. Conformal symmetry will reveal a result that would otherwise appear mysterious: we find in this setting of conformal field theory that the single-copy entanglement is just half the entropy of entanglement, in the leading contribution, i.e.,

$$
\lim _{L \rightarrow \infty} \frac{S\left(\rho_{L}\right)}{E_{1}\left(\rho_{L}\right)}=2 .
$$

In a single run, with a single invokation of a physical device acting on one system, one can obtain half the entanglement per specimen that is asymptotically available. This also gives a guideline how much entanglement one can expect to observe in actual single specimens of critical quantum systems.

This result also reveals an intriguing relationship between the largest eigenvalue of the reduction $\rho_{L}$ and its full spectrum of the reduction in a very large class of critical systems in the context of conformal field theory. These findings will be further substantiated by analogous results on a chain: for 
all translationally invariant quantum spin Hamiltonians that can be mapped onto isotropic quadratic fermionic Hamiltonians under Jordan-Wigner transformations [17], we find that if the entropy of a block is logarithmically divergent, so is the single-copy entanglement, with a factor of two difference in the prefactor. We finally check with the analytical example of the XY spin chain that, away from criticality, this simple relation between single-copy entanglement and entanglement entropy only holds close to the quantum phase transition point.

Exact conformal field theory computation. - Physical properties of quantum many-body systems are dictated at criticality by the underlying symmetry under scale transformations. If these systems are described by means of a quantum field theory setting, it can be seen that the underlying symmetry group is even larger and becomes the so-called conformal group of transformations. Many body systems on a lattice such as spin chains at a critical point are assessible by a conformal field theory that is invariant under the conformal group. In 1 spatial dimension, this group completely determines the physics of the system at hand [24]. A key role is played by the central charge $c$ of the system, the value of which will depend on the particular theory under consideration. Our result particularizes to a wide variety of quantum chains at criticality, such as the quantum XX spin model $(c=1)$, the critical quantum Ising model $(c=1 / 2)$ or the critical 3 -state Potts model $(c=4 / 5)$ [24]. In our setting, for a block of size $L$, conformal field theory provides us with an expression for the reduced density matrix, which describes the vacuum of the theory. We find [14, 24, 26]

$$
\rho_{L}=\frac{1}{Z_{L}(q)} q^{-c / 12} q^{\left(L_{0}+\bar{L}_{0}\right)},
$$

where $L_{0}$ and $\bar{L}_{0}$ are some positive semi-definite operators, $Z_{L}(q)=q^{-c / 12} \operatorname{tr}\left[q^{\left(L_{0}+\bar{L}_{0}\right)}\right]$ is the partition function on a torus, $q=e^{2 \pi i \tau}, \tau=(i \pi) /(\log (L / \epsilon))$ being the so-called modular parameter, $c$ is the central charge, and $\epsilon$ being a shortdistance cut-off which regularizes the theory. For the particularly important case of critical quantum chains $\epsilon=1$, corresponding to the lattice spacing of the chain [25]

The largest eigenvalue of the density matrix corresponds to the zero eigenvalue of $\left(L_{0}+\bar{L}_{0}\right)$, that is,

$$
\lambda_{1}=\frac{1}{Z_{L}(q)} q^{-c / 12}
$$

We then get for the single-copy entanglement $E_{1}\left(\rho_{L}\right)=$ $\log \left\lfloor 1 / \lambda_{1}\right\rfloor=\log \left\lfloor Z_{L}(q) q^{c / 12}\right\rfloor$. The leading behavior for the partition function can be computed when $L$ is large by taking advantage of its invariance under the so-called modular transformations [24, 27]. It is now possible to expand the partition function in powers of $\tilde{q}, \tilde{q}=e^{-2 \log L}$, as being done in Refs. [14, 26], and find that the leading contribution originates from the central charge $c, \log Z_{L}(\tilde{q})=-(c / 12) \log \tilde{q}+O(1 / L)=$ $(c / 6) \log L+O(1 / L)$. This result translates into an explicit expression for the single-copy entanglement

$$
E_{1}\left(\rho_{L}\right)=\frac{c}{6} \log L-\frac{c}{6} \frac{\pi^{2}}{\log L}+O(1 / L) .
$$

Eq. (4) is exact up to polynomial corrections in $1 / L$ since no further powers of $1 / \log L$ appear in the expansion [28].

Similar conformal field theory manipulations were used to show that the von Neumann entropy for the reduced density matrix is given by $S\left(\rho_{L}\right)=-(c / 6) \log \tilde{q}+O(1 / L)$ [26], which implies a direct relation between entropy and singlecopy entanglement

$$
E_{1}\left(\rho_{L}\right)=\frac{1}{2} S\left(\rho_{L}\right)-\frac{c}{6} \frac{\pi^{2}}{\log L}+O((1 / L) \log L),
$$

the last subleading correction being easily calculated from the results in Ref. [26]. This result fixes completely the value of the leading eigenvalue of the reduced density matrix of the block of size $L$ to be dictated by its entropy, that is, $\lim _{L \rightarrow \infty} \log \left(1 / \lambda_{1}\right) / S\left(\rho_{L}\right)=1 / 2$. Corrections to this limit can be obtained from Eq. (5). Quite remarkably, all the eigenvalues will inherit the same leading behavior and differ by their subleading corrections controlled by the positive eigenvalues of $\left(L_{0}+\bar{L}_{0}\right)$. This result establishes the quantitative connection between the single-copy entanglement and the geometric entropy in all critical systems that can be described in the framework of conformal field theory.

Spin chains corresponding to general quasi-free fermionic models. - We will aim at strenghening the previously achieved result by investigating the same question in a different setting: we will investigate all translationally invariant spin models that can, under a Jordan-Wigner transformation, be written as an isotropic quadratic Hamiltonian in fermionic operators. This setting includes the XX model.

The Jordan-Wigner transformation relates the Pauli operators in the spin system to fermionic operators obeying $\left\{c_{j}, c_{k}\right\}=0$ and $\left\{c_{j}^{\dagger}, c_{k}\right\}=\delta_{j, k}$, according to

$\sigma_{l}^{x}=\prod_{k<l} \sigma_{k}^{z}\left(c_{l}+c_{l}^{\dagger}\right), i \sigma_{l}^{y}=\prod_{k<l} \sigma_{k}^{z}\left(c_{l}-\hat{c}_{l}^{\dagger}\right), \sigma_{l}^{z}=1-2 c_{l}^{\dagger} c_{l}$.

The ground state is a quasi-free fermionic state, so a state that is completely characterized by the second moments of fermionic operators. Consider now such an infinite spin chain that corresponds to a general translationally invariant isotropic quasi-free fermionic model. These embody chain systems the Hamiltonian of which can be cast into the form $H=\sum_{l, k} c_{l}^{\dagger} A_{l-k} c_{k}$, with some general $A_{l}=A_{-l} \in \mathbb{R}$ of which we do not make an assumption. The statement we arrive at is the following: if the entropy satisfies

$$
S\left(\rho_{L}\right)=\xi \log L+O(1)
$$

for some $\xi>0$, then the single-copy entanglement satisfies

$$
E_{1}\left(\rho_{L}\right)=\frac{1}{2} S\left(\rho_{L}\right)+O(1)
$$

That is, if we find that the entropy of entanglement scales asymptotically as the logarithm of $L-$ as encountered in this class of systems exactly at criticality - then we can infer that the single-copy entanglement will be asymptotically exactly one half of it, in the leading order terms. This does notably 
not fix such a relationship in case that, for example, the system is gapped and the entropy of entanglement saturates. This statement follows from the subsequent argument.

The reduced state of a block of length $L$ is entirely specified by the eigenvalues of the real symmetric $L \times L$ Toeplitz matrix $T_{L}$, which defines the second moments of fermionic operators. The fact that $T_{L}$ is a Toeplitz matrix reflects the translational invariance of the model, being symmetic follows from the isotropy. The $l$-th row of this matrix is given by $\left(t_{-l+1}, t_{-l+2}, \ldots, t_{0}, \ldots, t_{L-l}\right)$ [30]. The latter represents the Fermi surface, and essentially characterizes the fermionic model. The eigenvalues of $T_{L}$ will be labeled as $\mu_{1}, \ldots \mu_{L} \in$ $[-1,1]$, which are the zeros of the characteristic polynomial $F: \mathbb{C} \rightarrow \mathbb{C}, F(z)=\operatorname{det}\left[z \mathbb{I}_{L}-T_{L}\right]$. This function $F$ is meromorphic, and all its real zeros are contained in the interval $[-1,1]$, corresponding to the spectrum of $T_{L}$. The entropy of entanglement can be obtained as $S\left(\rho_{L}\right)=\sum_{l=1}^{L} f_{S}\left(1, \mu_{l}\right)$ [9, 11], where $f_{S}: \mathbb{R}^{+} \times \mathbb{C} \rightarrow \mathbb{C}$ as a complex embedding is defined as $f_{S}(x, y)=-((x+y) / 2) \log ((x+y) / 2)-((x-$ $y) / 2) \log ((x-y) / 2)$ to avoid problems with non-analyticities. Actually, we can write [9, 11]

$$
S\left(\rho_{L}\right)=\lim _{\varepsilon \searrow 0} \lim _{\delta \searrow 0} \frac{1}{2 \pi i} \int f_{S}(1+\varepsilon, z) \frac{F^{\prime}(z)}{F(z)} d z .
$$

The contour of the integration is shown in Fig. 11 which is as in Ref. [16], but slightly different from the one in Ref. [9]. In turn, we may write $-\log \lambda_{1}=\sum_{l=1}^{L} f_{1}\left(0, \mu_{l}\right)$ [16], in terms of the above $\mu_{1}, \ldots, \mu_{L}$, where now $f_{1}: \mathbb{R}^{+} \times \mathbb{C} \rightarrow \mathbb{C}$, $f_{1}(\varepsilon, z)=-\log \left(\left(1+\left(z^{2}+\varepsilon^{2}\right)^{1 / 2}\right) / 2\right)$. Respecting the cuts of the logarithm (see Refs. [16] and [33]), we may cast $-\log \lambda_{1}$ and hence (up to integer brackets) $E_{1}\left(\rho_{L}\right)$ into the form

$$
-\log \lambda_{1}=\lim _{\varepsilon \searrow 0} \lim _{\delta \searrow 0} \frac{1}{2 \pi i} \int f_{1}(\varepsilon, z) \frac{F^{\prime}(z)}{F(z)} d z .
$$

Now we know that $T_{L}$ is a real symmetric Toeplitz matrix, which means that we can assess the asymptotic behavior of their determinants. This can be done using proven instances of the Fisher-Hartwig conjecture [11, 32]; proven instances, as we consider isotropic models [11]. Concerning the function $F: \mathbb{C} \rightarrow \mathbb{C}$, this observation enables us to write

$$
\frac{F^{\prime}(z)}{F(z)}=a(z) L-b(z) \log L+O(1)
$$

where $b(z)=-2 R \beta(z) \beta^{\prime}(z)$, with $\beta: \mathbb{C} \rightarrow \mathbb{C}$ being a function defined as $\beta(z)=\log ((z+1) /(z-1)) /(2 \pi i)$, see Refs. [11]. $R$ in turn is half the number of discontinuities of the above symbol in the interval $[0,2 \pi)$. For the XX model, e.g., we have that $R=1$. Now, if Eq. (7) is valid, then

$$
\lim _{\varepsilon \searrow 0} \lim _{\delta \searrow 0} \int f_{S}(1+\varepsilon, z) a(z) d z=0
$$

But since $S\left(\rho_{L}\right) \geq E_{1}\left(\rho_{L}\right)$ for all $L \in \mathbb{N}$, necessarily $\lim _{\varepsilon \searrow 0} \lim _{\delta \searrow 0} \int f_{1}(\varepsilon, z) a(z) d z=0$ must hold. Hence, we only have to consider the logarithmically divergent term. It is

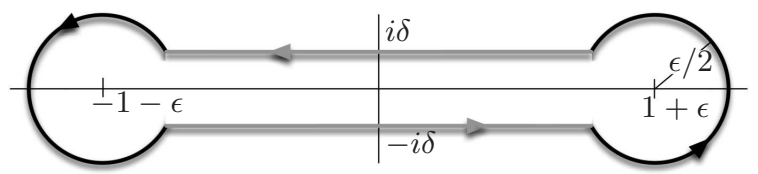

FIG. 1: Contour of integration to be taken in case of both the entropy of entanglement and the single-copy entanglement.

sufficient for our argument, therefore, for the entropy of entanglement and for the single-copy entanglement to consider the contour integrals

$$
\begin{aligned}
I_{S} & =\lim _{\varepsilon \searrow 0} \lim _{\delta \searrow 0} \frac{1}{2 \pi i} \int f_{S}(1+\varepsilon, z) b(z) d z, \\
I_{1} & =\lim _{\varepsilon \searrow 0} \lim _{\delta \searrow 0} \frac{1}{2 \pi i} \int f_{1}(\varepsilon, z) b(z) d z .
\end{aligned}
$$

$b$ is analytic outside $[-1,1]$. In turn, this means that the contributions of the circle pieces vanish in both cases. Hence, we finally arrive at

$$
\begin{aligned}
S\left(\rho_{L}\right) & =\frac{R}{\pi^{2}} \int_{-1}^{1} d x \frac{f_{S}(1, x)}{1-x^{2}} \log L+O(1), \\
E_{1}\left(\rho_{L}\right) & =\frac{R}{\pi^{2}} \int_{-1}^{1} d x \frac{f_{1}(0, x)}{1-x^{2}} \log L+O(1) .
\end{aligned}
$$

Since $f_{1}(0, x)=-\log (1+|x|) / 2$ for $x \in[-1,1]$, this gives

$$
S=\frac{R}{3} \log L+O(1), E_{1}=\frac{R}{6} \log L+O(1),
$$

which in turn implies the validity of Eq. 8): In these models, whenever the system is critical, the single-copy entanglement is again exactly half the asymptotically available in its leading contribution. This gives further substance to the previous consideration in a language of conformal field theory.

Single-copy entanglement away from criticality. - The relation between single-copy entanglement and entropy can be demonstrated near critical points in some integrable models. We illustrate this fact using the XY spin chain, in a slightly different set-up: we consider the chain of length $N$ with periodic boundary conditions, where the half chain $L=N / 2$ constitutes one system. For large $N$, the density matrix of the system can be arbitrarily well approximated in trace-norm by

$$
\frac{e^{-H}}{\operatorname{tr}\left[e^{-H}\right]}, H=\sum_{k} \epsilon_{k} d_{k}^{\dagger} d_{k}, \epsilon_{k}= \begin{cases}2 k \epsilon, & \text { if } \lambda<1 \\ (2 k+1) \epsilon, & \text { if } \lambda>1\end{cases}
$$

[6]. Here, $k \in \mathbb{N}, \lambda \in \mathbb{R}$ is the parameter controlling the external magnetic field, $\lambda^{*}=1$ corresponds to the quantum phase transition point, and $\epsilon=\pi\left(I\left(\left(1-x^{2}\right)^{1 / 2}\right)\right) / I(x)$, $I: \mathbb{C} \rightarrow \mathbb{C}$ is the complete elliptic integral of the first kind, $I(x)=\int_{0}^{\pi / 2} d \theta /\left(1-x^{2} \sin ^{2}(\theta)\right)^{1 / 2} . x$ is related to $\lambda$ and $\gamma:$

$$
x= \begin{cases}\left(\lambda^{2}+\gamma^{2}-1\right)^{1 / 2} / \gamma, & \text { if } \lambda<1 \\ \gamma /\left(\lambda^{2}+\gamma^{2}-1\right)^{1 / 2}, & \text { if } \lambda>1\end{cases}
$$


with the condition $\lambda^{2}+\gamma^{2}>1$ (external region of the BMcircle [32]). A computation of the single-copy entanglement with respect to this partitioning can be performed in terms of $\epsilon$, transforming sums into integrals by means of the EulerMcLaurin expansion, and finding

$$
-\log \lambda_{1}= \begin{cases}\frac{\pi^{2}}{24 \epsilon}-\frac{\epsilon}{24}+O\left(e^{-\epsilon}\right) & \text { if } \lambda<1 \\ \frac{\pi^{2}}{24 \epsilon}+\frac{\log 2}{2}+\frac{\epsilon}{12}+O\left(e^{-\epsilon}\right) & \text { if } \lambda>1\end{cases}
$$

No subleading corrections in powers of $\epsilon$ do appear in the expansion. On the other hand it can be seen by explicit evaluation that that the entropy of the reduction $\rho_{L, \epsilon}$ can be related in this case to the single copy-entanglement by $S\left(\rho_{L, \epsilon}\right)=$ $-\left(1-\epsilon \frac{\partial}{\partial \epsilon}\right) \log \lambda_{1}$, which shows that

$$
\lim _{\epsilon \rightarrow 0} \frac{E_{1}\left(\rho_{L, \epsilon}\right)}{S\left(\rho_{L, \epsilon}\right)}=\frac{1}{2} .
$$

This is precisely the limit where the theory becomes critical.

In this work we have shown that the leading critical scaling of the single-copy entanglement is exactly one half of the entropy of entanglement in critical quantum spin chains, using tools of conformal field theory. We have also provided an analysis for all translationally-invariant quantum spin chains that can be mapped onto an isotropic quasi-free fermionic model under a Jordan-Wigner transformation, leading to similar conclusions. Away from criticality, this simple relation is recovered when approaching the quantum phase transition point, as seen in the XY model. It is a fact that the single-copy entanglement could be experimentally studied in, for instance, systems of cold atoms in optical lattices, ions in ion-traps, or solid-state devices. Our hope is that the results we have presented here will serve as guideline for that kind of experiments, as well as for a better understanding of the structure of the ground state correlations in quantum spin chains.

Acknowledgements. - Two of us (JE and JIL) would like to thank the members of the Perimeter Institute for Theoretical Physics - where part of the work was carried out - in particular D. Gottesman and M. Mosca for kind hospitality. We would like to thank P. Calabrese, J.I. Cirac, V.E. Korepin, T. Osborne, M.B. Plenio, R.F. Werner, and M.M. Wolf for discussions. This work has been supported by the DFG (SPP 1116, SPP 1078), the EU (QUPRODIS, QAP), the EPSRC, the EURYI scheme, and the MEC (Spain).

Note added: After completion of this work, we became aware of the independent work Ref. [34], where the first (leading-order) term for single-copy entanglement in the conformal case was also discussed in detail and clarity.
[1] A. Osterloh, L. Amico, G. Falci, and R. Fazio, Nature 416, 608 (2002); T.J. Osborne and M.A. Nielsen, Phys. Rev. A 66, 032110 (2002).

[2] K. Audenaert, J. Eisert, M.B. Plenio, and R.F. Werner, Phys. Rev. A 66, 042327 (2002); A. Botero and B. Reznik, Phys. Rev. A 70, 052329 (2004).

[3] G. Vidal, J.I. Latorre, E. Rico, and A. Kitaev, Phys. Rev. Lett. 90, 227902 (2003).

[4] M. Fannes, B. Haegeman, and M. Mosonyi, J. Math. Phys. 44, 6005 (2003); S. Farkas and Z. Zimboras, J. Math. Phys. 46, 123301 (2005).

[5] P. Calabrese and J. Cardy, J. Stat. Mech. 06, 002 (2004); P. Calabrese and J. Cardy, quant-ph/0505193

[6] I. Peschel, J. Stat. Mech. - Th. E P12005 (2004).

[7] J.I. Latorre, E. Rico, and G. Vidal, Quant. Inf. Comp. 4, 48 (2004).

[8] M. Hein, J. Eisert, and H.J. Briegel, Phys. Rev. A 69, 062311 (2004).

[9] B.-Q. Jin and V.E. Korepin, J. Stat. Phys. 116, 79 (2004).

[10] M.B. Plenio, J. Eisert, J. Dreissig, and M. Cramer, Phys. Rev. Lett. 94, 060503 (2005); M. Cramer, J. Eisert, M.B. Plenio, and J. Dreissig, Phys. Rev. A 73, 012309 (2006).

[11] A.R. Its, B.-Q. Jin, and V.E. Korepin, J. Phys. A 38, 2975 (2005); J.P. Keating and F. Mezzadri, Phys. Rev. Lett. 94, 050501 (2005).

[12] M.M. Wolf, Phys. Rev. Lett. 96, 010404 (2006) ; D. Gioev and I. Klich, ibid. 96, 100503 (2006).

[13] J.I. Latorre, C.A. Lütken, E. Rico, and G. Vidal, Phys. Rev. A 71, 034301 (2005).

[14] R. Orus, Phys. Rev. A 71, 052327 (2005).

[15] F. Verstraete and J.I. Cirac, cond-mat/0505140

[16] J. Eisert and M. Cramer, Phys. Rev. A 72, 042112 (2005)
[17] S. Sachdev, Quantum Phase Transitions (Cambridge University Press, Cambridge, 1999).

[18] C.H. Bennett et al, Phys. Rev. A 53, 2046 (1996).

[19] Note that $|i, j\rangle, i, j=1, \ldots, M$, are elements of a product basis with respect to the distinguished block and the rest of the chain.

[20] M.A. Nielsen, Phys. Rev. Lett. 83, 436 (1999).

[21] In a probabilistic version $E_{p}$, one may also allow for maximally entangled states of different dimensions with certain probabilities, compare Ref. [23].

[22] We define the entropy, as well as the single-copy entanglement, in terms of the natural logarithm.

[23] D. Jonathan and M.B. Plenio, Phys. Rev. Lett. 83, 1455 (1999).

[24] P. Ginsparg, in Applied Conformal Field Theory (Les Houches Summer School, France, 1988).

[25] This value of the cut-off is to be understood in all the forthcoming calculations.

[26] C. Holzhey, F. Larsen, and F. Wilczek, Nucl. Phys. B 424, 443 (1994).

[27] The needed transformation is $\tau \mapsto-1 / \tau$, which amounts to $Z_{L}(q)=Z_{L}(\tilde{q})$, with $q=e^{-2 \pi^{2} / \log L}$ and $\tilde{q}=e^{-2 \log L}$.

[28] Note that the leading order term also follows from the results on the expressions for the Renyi entropies [31] in the conformal setting in Ref. [5]. The non-leading term can be explicitely checked by means of numerical evaluations or analytical derivations for integrable models like the one in Ref. [29].

[29] I. Peschel, J. Stat. Mech. P06004 (2004).

[30] These numbers are for an infinite chain found to be $t_{l}=$ $\int_{0}^{2 \pi} g(k) e^{-i l k} d k /(2 \pi)$, where $g: \mathbb{C} \rightarrow \mathbb{C}$ is the symbol [32].

[31] P. Calabrese, private communication (2005).

[32] E. Lieb, T. Schultz, and D. Mattis, Ann. Phys. 16, 407 (1961); E. Barouch and B.M. McCoy, Phys. Rev. A 3, 786 (1971); T. Ehrhardt and B. Silbermann, Funct. Anal. 148, 229 (1997). 
[33] For $x=|x| e^{i \varphi} \in \mathbb{C} \backslash\{z \in \mathbb{C}: \Im(z)=0, \Re(z)<0\}$ we take $\log x=\log |x|+i \varphi$.
[34] I. Peschel and J. Zhao, J. Stat. Mech. P11002 (2005). 